\title{
Familles Knesériennes et le Problème aux Limites, II
}

\author{
Par \\ Masuo Hukuhara
}

\section{Résultats de H.-W. Knobloch}

Concernant l'équation différentielle ordinaire

$$
d^{2} x / d t^{2}=f(t, x, d x / d t)
$$

Knobloch a obtenu des théorèmes d'existence qui coïncident à peu près avec ceux de Nagumo. Nous voulons montrer que ses résultats se déduisent aussi de notre théorie.

Soit $I$ un intervalle compact, qui peut être supposé $[0,1]$ sans perdre la généralité. $I$ est décomposé en un nombre fini d'intervalles $\left[t_{i}, t_{i+1}\right]$. Soit $\mathscr{D}$ un compact dans $I \times R^{2}$ et posons

$$
\mathscr{D}_{i}=\left\{(t, x, y) \in \mathscr{D} ; t \in\left[t_{i}, t_{i+1}\right]\right\} .
$$

Knobloch a supposé que

(i ) $f \in \mathrm{C}\left(\mathscr{D}_{i}\right)$;

(ii) $f(t, x, y)$ satisfait à la condition de Lipschitz par rapport à $x$ et $y$ dans chaque $\mathscr{D}_{i}$.

Knobloch prend pour le domaine $\mathscr{D}$ où $f$ est définie le domaine non borné $\mathscr{D} \times R^{1}$. Nous prenons pour $\mathscr{D}$ le domaine compact

$$
\mathscr{D}=\{(t, x, y) ;(t, x) \in \mathfrak{D}, \underline{\Omega}(t, x) \leqq y \leqq \bar{\Omega}(t, x)\} .
$$

Car notre théorie n'utilise point les valeurs de $f$ en des points où l'on a $y<\underline{\Omega}(t, x)$ ou $y<\bar{\Omega}(t, x)$.

La condition (i) est évidemment plus générale que la condition

Received March 11, 1968. 
$f \in \mathrm{C}(\mathscr{D})$. Mais cette généralisation n'est pas importante. On pourra généraliser encore; par exemple on pourra traiter de la même manière l'équation du type de Carathéodory. Dans la suite nous supposerons pour la simplicité que $f \in \mathrm{C}(\mathscr{D})$.

Quant à la condition (ii), elle est une des conditions d'unicité. Elle joue un rôle important lorsqu'on montre l'existence d'une solution jouissant d'une propriété que Knobloch appelle propriété (B). Mais s'il ne s'agit pas de cette propriété, il me semble que la condition ne joue aucun rôle essentiel. Pourtant pour le voir, il serait nécessaire de faire quelques études préliminaires sur les applications semi-continues supérieurement à valeurs compactes.

\section{Rappel des Résultats de Knobloch.}

Citons quelques-unes des propositions que Knobloch a obtenues.

Soient

$$
\begin{aligned}
& \mathscr{D}=\{(t, x) ; t \in I, \underline{\omega}(t) \leqq x \leqq \bar{\omega}(t)\}, \\
& \mathscr{D}=\{(t, x, y) ;(t, x) \in \mathfrak{D}, \underline{\Omega}(t, x) \leqq y \leqq \bar{\Omega}(t, x)\},
\end{aligned}
$$

où $\underline{\omega}$ et $\bar{\omega}$ sont des fonctions $\mathrm{C}^{2}[\mathrm{I}]$ telles que l'on ait

$$
\left\{\begin{array}{l}
\underline{\omega}^{\prime \prime}(t) \geqq f\left(t, \underline{\omega}(t), \underline{\omega}^{\prime}(t)\right), \\
\bar{\omega}^{\prime \prime}(t) \leqq f\left(t, \bar{\omega}(t), \bar{\omega}^{\prime}(t)\right)
\end{array}\right.
$$

dans $I$, et $\underline{\Omega}$ et $\bar{\Omega}$ sont des fonctions $\mathrm{C}^{1}[\mathfrak{D}]$ telles que

$$
f(t, x, \Omega)-\Omega \Omega_{x}-\Omega_{t}
$$

garde une signe constante dans $\mathfrak{D}=D-E$ pour $\Omega=\underline{\Omega}$, $\bar{\Omega}$, où

$$
E=\{(t, x) \in \mathfrak{D} ; \underline{\Omega}(t, x) \geqq \bar{\Omega}(t, x)\} .
$$

Proposition 1. Si l'on a

$$
\underline{\omega}(t)<\bar{\omega}(t)
$$

pour $t \in I$,

$$
\underline{\Omega}(t, x)<\bar{\Omega}(t, x)
$$


pour $t=0,1, \underline{\omega}(t) \leqq x \leqq \bar{\omega}(t)$ et

$$
\left\{\begin{array}{l}
\underline{\Omega}(t, \underline{\omega}(t))<\underline{\omega}^{\prime}(t)<\bar{\Omega}(t, \underline{\omega}(t)), \\
\underline{\Omega}(t, \bar{\omega}(t))<\bar{\omega}^{\prime}(t)<\bar{\Omega}(t, \bar{\omega}(t))
\end{array}\right.
$$

pour $t=0,1$, l'équation (1) admet une solution $\varphi$ satisfaisant aux inégalités

$$
\begin{gathered}
\underline{\omega}(t)<\varphi(t)<\bar{\omega}(t), \\
\underline{\Omega}(t, \varphi(t))<\varphi^{\prime}(t)<\bar{\Omega}(t, \varphi(t))
\end{gathered}
$$

pour $t \in I$.

Une fonction $\varphi$ est dite périodique par rapport à $I$ si $\varphi$ et $\varphi^{\prime}$ prennent les mêmes valeurs aux extrémités de $I$. Dans un de ses articles antérieurs Knobloch a obtenu un théorème d'existence, dont l'énoncé voici:

\section{Proposition 2. Supposons remplies les inégalités}

$$
\begin{gathered}
\underline{\omega}(t) \leqq \bar{\omega}(t), \\
\left\{\begin{array}{l}
\underline{\Omega}(t, \underline{\omega}(t)) \leqq \underline{\omega}^{\prime}(t) \leqq \bar{\Omega}(t, \underline{\omega}(t)), \\
\underline{\Omega}(t, \bar{\omega}(t)) \leqq \bar{\omega}^{\prime}(t) \leqq \bar{\Omega}(t, \bar{\omega}(t))
\end{array}\right.
\end{gathered}
$$

pour $t \in I$ et la condition de périodicité

$$
\begin{gathered}
\underline{\omega}(0)=\underline{\omega}(1), \bar{\omega}(0)=\bar{\omega}(1), \\
\underline{\Omega}(0, x)=\underline{\Omega}(1, x), \bar{\Omega}(0, x)=\bar{\Omega}(1, x) .
\end{gathered}
$$

L'équation (1) admet alors une solution périodique par rapport à I.

M. Nagumo n'a pas traité la condition de périodicité. Nous voulons montrer comment on peut traiter la condition de périodicité à l'aide de notre théorie.

En utilisant la proposition 2, Knobloch a établi un théorème d'existence de solutions ayant une propriété qu'il a appelée propriété (B).

Par définition, une solution $\varphi$ jouit de la propriété (B) s'il existe une suite de solutions $\left\{\varphi_{\nu}\right\}$ telles que

$\left(\mathrm{B}_{1}\right) \quad \varphi_{\nu} \rightarrow \varphi, \varphi_{\nu}^{\prime} \rightarrow \varphi^{\prime}$ uniformément dans $I$; 
$\left(\mathrm{B}_{2}\right)$ la différence $\Delta_{\nu}=\varphi-\varphi_{\nu} \neq 0$ a la même signe pour tout nombre naturel $\nu$ et toute valeur $t \in I$;

$\left(\mathrm{B}_{3}\right) \quad\left|\Delta_{\nu}^{\prime}\right| \leqq c\left|\Delta_{\nu}\right|$ pour tout nombre naturel $\nu$ et toute valeur $t \in I$ avec une constante $c$ indépendante de $\nu$ et de $t$.

Le théorème d'existence s'énonce alors comme suit.

Proposition 3. Supposons remplies toutes les hypothèses de la proposition I. Il existe alors une solution $\varphi$ jouissant de la propriété (B) et satisfaisant aux inégalités

$$
\begin{gathered}
\underline{\omega}(t) \leqq \varphi(t) \leqq \bar{\omega}(t), \\
\underline{\Omega}(t, \underline{\omega}(t)) \leqq \varphi^{\prime}(t) \leqq \bar{\Omega}(t, \bar{\omega}(t))
\end{gathered}
$$

pour $t \in I$.

Nous montrerons que l'on peut remplacer les inégalités (13.12), (13.13) par les inégalités strictes $(13.6),(13.7)$ et la condition $\left(B_{3}\right)$ par la condition plus forte:

$\left(\mathrm{B}_{3}^{\prime}\right) \quad$ On a

$$
\sup \left\{\left|\Delta_{\nu}^{\prime}(t)\right|, t \in I\right\} \leqq c \inf \left\{\left|\Delta_{\nu}(t)\right| ; t \in I\right\}
$$

pour tout nombre naturel $\nu$ et toute valeur $t \in I$ avec une constante $c$ indépendante de $\nu$ et de $t$.

\section{Réflexions sur les Inégalités Relatives à $\omega$ et $\mathscr{8}$.}

Considérons par exemple le cas où l'expression (13.2) est positive pour $\Omega=\underline{\Omega}$ et supposons l'existence des valeurs $t \in\langle 0,1\rangle$ telles que l'on ait

$$
\underline{\omega}^{\prime}(t) \leqq \underline{\Omega}(t, \underline{\omega}(t)) \text {. }
$$

Si $\tau$ est la borne inférieure de telles valeurs, on a l'inégalité

$$
\underline{\omega}^{\prime}(t)>\underline{\Omega}(t, \underline{\omega}(t)) \quad \text { pour } \quad 0 \leqq t<\tau
$$

et l'égalité

$$
\underline{\omega}^{\prime}(\tau)=\underline{\Omega}(\tau, \underline{\omega}(\tau)) \text {. }
$$


Si donc on pose $\xi=\underline{\omega}(\tau), \eta=\underline{\omega^{\prime}}(\tau)$, on a

$$
\begin{aligned}
\underline{\omega}^{\prime \prime}(\tau) & \leqq \Omega_{t}(\tau, \xi)+\underline{\Omega}(\tau, \xi) \underline{\Omega}_{x}(\tau, \xi) \\
& <f(\tau, \xi, \eta) \leqq \underline{\omega}^{\prime \prime}(\tau) .
\end{aligned}
$$

On obtient ainsi une impossibilité. L’inégalité

$$
\underline{\omega}^{\prime}(t)>\underline{\Omega}(t, \underline{\omega}(t))
$$

subsiste donc dans tout l'intervalle.

Par conséquent, les inégalités (13.5) sont vérifiées non seulement pour $t=0,1$ mais même pour toutes les valeurs $t \in I$.

Si l'ensemble $E$ n'est pas vide, il est fermé. Soit $\tau$ la plus petite des abscisses des points de $E$. Un point $(\tau, \zeta) \in E$ appartient à l'intérieur de $\mathfrak{D}$ et on a nécessairement

$$
\underline{\Omega}(\tau, \xi)=\bar{\Omega}(\tau, \xi), \underline{\Omega}_{t}(\tau, \xi) \geqq \bar{\Omega}_{t}(\tau, \xi), \underline{\Omega}_{x}(\tau, \xi)=\bar{\Omega}_{\lambda}(\tau, \xi) .
$$

On a donc

$$
\begin{array}{r}
f(\tau, \xi, \underline{\Omega}(\tau, \xi))-\underline{\Omega}(\tau, \xi) \underline{\Omega}_{x}(\tau, \xi)-\underline{\Omega}_{l}(\tau, \xi) \\
\leqq f(\tau, \xi, \bar{\Omega}(\tau, \xi))-\bar{\Omega}(\tau, \xi) \bar{\Omega}_{x}(\tau, \xi)-\bar{\Omega}_{t}(\tau, \xi) .
\end{array}
$$

On ne peut avoir cette inégalité si l'expression (13.2) est positive pour $\Omega=\underline{\Omega}$ et négative pour $\Omega=\bar{\Omega}$. L'ensmeble $E$ est donc vide.

Par conséquent, si l'expression (13.2) est positive pour $\Omega=\underline{\Omega}$ et négative pour $\Omega=\bar{\Omega}$, on a l'inégalité (13.4) dans $D$. Il en est de même du cas où l'expression (13.2) est négative pour $\Omega=\underline{\Omega}$ et positive pour $\Omega=\bar{\Omega}$.

Si l'expression $\Omega$ a le même signe pour $\Omega=\underline{\Omega}, \bar{\Omega}$, l'ensemble $E$ peut être non vide, auquel cas il se trouve à l'intérieur du domaine D limité par les quatre lignes

$$
\begin{aligned}
& 0 \leqq t \leqq 1, x=\underline{\omega}(t) ; 0 \leqq t \leqq 1, x=\bar{\omega}(t) ; \\
& t=0, \underline{\omega}(0) \leqq x \leqq \bar{\omega}(0) ; t=1, \underline{\omega}(1) \leqq x \leqq \bar{\omega}(1) .
\end{aligned}
$$

La frontière de $D$ se compose de ces quatre lignes et de la frontière de $E$. 


\section{Démonstration de la Proposition 1 .}

Soit $\mathbf{F}$ l'ensemble des solutions du système différentiel équivalent à l'équation (1):

$$
d x / d t=y, d y / d t=f(t, x, y) .
$$

Comme nous avons remarqué dans notre article précédent, tous les points de $\mathscr{D}$ sont knesériens sauf les points $(t, x)$ où l'on a l'égalité

$$
\underline{\Omega}(t, x)=\bar{\Omega}(t, x) \text {. }
$$

Mais on voit sans peine que si l'on a cette égalité, le point $(t, x)$ appartient à l'intersection $\mathscr{B}^{d} \cap \mathscr{B}^{s}$.

Par suite, $\mathbf{F}$ est une famille knesérienne dont le domaine fondamental est $\mathscr{D}$.

Considérons d'abord le cas où l'expression (13.2) est positive pour $\Omega=\underline{\Omega}$ et négative pour $\Omega=\bar{\Omega}$.

La section $\mathcal{S}_{0}$ de $\mathscr{D}$ par le plan $t=0$ est évidemment contenue dans $\mathscr{B}^{g}$. Les points de $\mathscr{B}^{d}$ dans $\mathcal{S}_{0}$ forment deux segments

$$
\begin{array}{ll}
\mathrm{BC}: & x=\underline{\omega}(0), \underline{\Omega}(0, \underline{\omega}(0)) \leqq y<\omega^{\prime}(0) ; \\
\mathrm{EF}: & x=\bar{\omega}(0), \bar{\omega}^{\prime}(1)<y \leqq \bar{\Omega}(1, \bar{\omega}(1)) .
\end{array}
$$

Les points $\mathrm{B}$ et $\mathrm{H}$ appartiennent à $\mathscr{B}^{d} \cup \mathscr{B}_{+}$et les autres points de la section appartiennent à $\mathscr{B}^{+}$.

La section $\mathcal{S}_{1}$ de $\mathscr{D}$ par le plan $t=1$ est évidemment contenue dans $\mathscr{B}^{d}$. Les points de $\mathscr{B}^{g}$ forment deux segments

$$
\begin{array}{ll}
\mathrm{AB}: & x=\underline{\omega}(1), \underline{\underline{\omega}}(1)<y \leqq \bar{\Omega}(1, \underline{\omega}(1)) ; \\
\mathrm{DE}: & x=\bar{\omega}(1), \underline{\Omega}(1, \bar{\omega}(1)) \leqq y<\bar{\omega}^{\prime}(1) .
\end{array}
$$

Les points $\mathrm{B}$ et $\mathrm{E}$ appartiennent à $\mathscr{B}^{g} \cup \mathscr{B}_{\text {_ }}$ et les autres points de la section appartiennent à $\mathscr{B}^{-}$.

Dans la section $\mathcal{S}_{\tau}$ de $\mathscr{D}$ par le plan $t=\tau(0<\tau<1), \mathscr{B}^{g} \cup \mathscr{B}_{-}$se compose de deux parties

$$
\mathrm{FAB}: \quad\left\{\begin{array}{l}
\underline{\omega}(\tau) \leqq x \leqq \bar{\omega}(\tau), y=\bar{\Omega}(\tau, x) ; \\
x=\underline{\omega}(\tau), \underline{\omega}^{\prime}(\tau) \leqq y \leqq \bar{\Omega}(\tau, \bar{\omega}(\tau)) ;
\end{array}\right.
$$


$\mathrm{CDE}: \quad\left\{\begin{array}{l}\underline{\omega}(\tau) \leqq x \leqq \bar{\omega}(\tau), y=\underline{\Omega}(\tau, x) ; \\ \left.x=\bar{\omega}(\tau), \underline{\Omega}(\tau, \bar{\omega}(\tau)) \leqq y \leqq \bar{\omega}^{\prime} / \tau\right) ;\end{array}\right.$

$\mathscr{B}^{d} \cup \mathscr{B}_{+}$se compose de deux segments

$\mathrm{BC}: \quad x=\underline{\omega}(\tau), \underline{\Omega}(\tau, \underline{\omega}(\tau)) \leqq y \leqq \underline{\omega}^{\prime}(\tau) ;$

$\mathrm{EF}: \quad x=\bar{\omega}(\tau), \bar{\omega}^{\prime}(\tau) \leqq y \leqq \bar{\Omega}(\tau, \bar{\omega}(\tau))$.

Prenons pour $\mathcal{E}$ une courbe $\mathrm{GH}$ joignant les segments $\mathrm{BC}$ et $\mathrm{EF}$ dans la section $\mathcal{S}_{0}$ et pour $\mathcal{E}^{\prime}$ une courbe $\mathrm{KL}$ joignant les segments $\mathrm{AB}$ et $\mathrm{DE}$ dans la section $\mathcal{S}_{1}$.

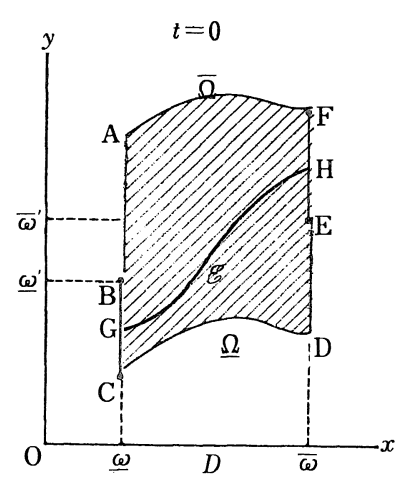

$\mathscr{B}^{d} \cup \mathscr{B}_{+}$

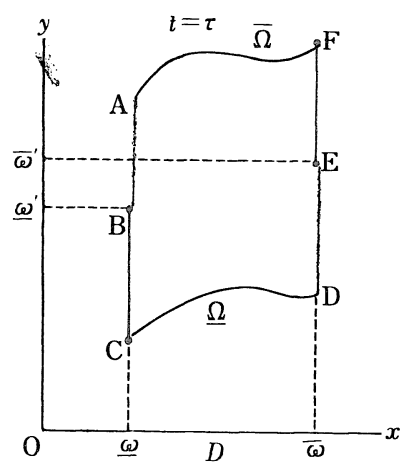

Fig. 1, 2, 3 .

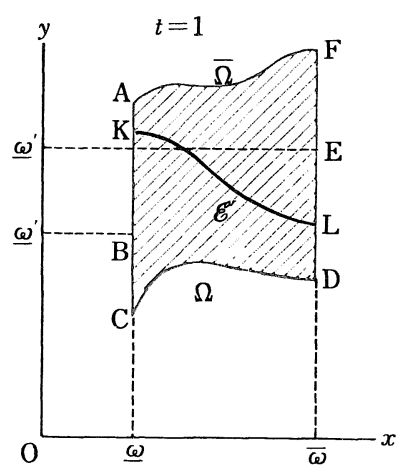

$\mathscr{B}^{+}$

On pourra appliquer notre théorème d'existence 9.1. F contient une courbe $x=\varphi(t), y=\varphi^{\prime}(t)$, qui joint $\mathcal{E}$ et $\mathcal{E}^{\prime}$. L'extrémité gauche de cette courbe ne peut coïncider avec $\mathrm{G}$ ni avec $\mathrm{H}$, car $\mathrm{G}$ et $\mathrm{H}$ appartienne à $\mathscr{B}^{d}$. On a par suite les inégalités strictes (13.6) pour $t=0$. On a de même ces inégalités pour $t=1$.

Jusqu'ici nous n'avons pas utilisé la condition d'unicité (ii). On peut donc affirmer, sans cette hypothèse, l'existence d'une solution $\varphi$ telle que l'on ait

$$
\underline{\omega}(t) \leqq \varphi(t) \leqq \bar{\omega}(t)
$$

dans $I$, les inégalités strictes ayant lieu aux extrémités. 
$\varphi$ satisfaisant à la condition de $\underline{\omega}$, il existe une solution $\psi$ telle que l'on ait

$$
\varphi(t) \leqq \psi(t) \leqq \bar{\omega}(t)
$$

dans $I$, les inégalités strictes ayant lieu aux extrémités. Si l'on a l'égalité $\varphi(\tau)=\bar{\omega}(\tau)$ en une valeur $\tau$, on aura nécessairement

$$
\varphi(\tau)=\psi(\tau)=\bar{\omega}(\tau), \varphi^{\prime}(\tau)=\psi^{\prime}(\tau)=\bar{\omega}^{\prime}(\tau) .
$$

Si donc on suppose la condition d'unicité (ii), on doit avoir $\varphi(\tau)<$ $\bar{\omega}(\tau)$.

Par conséquent, si l'on suppose la condition d'unicité (ii), on a les inégalités (13.6).

C.Q.F.D.

On pourra traiter les autres cas sans aucune modification essentielle quoique l'ensemble $E$ défini au $\mathrm{n}^{\circ} 14$ ne soit pas vide si l'expression (13.2) a le même signe pour $\Omega=\underline{\Omega}$ et $\Omega=\bar{\Omega}$.

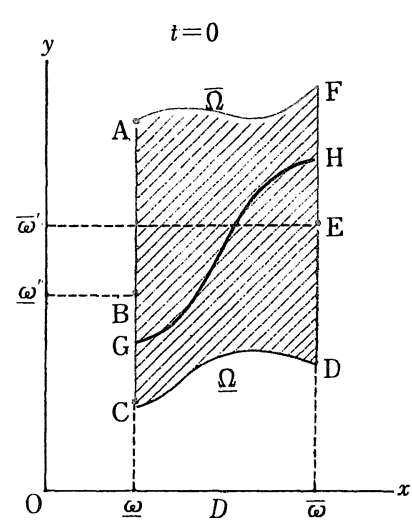

$\mathscr{B}^{d} \cup \mathcal{B}_{+}$
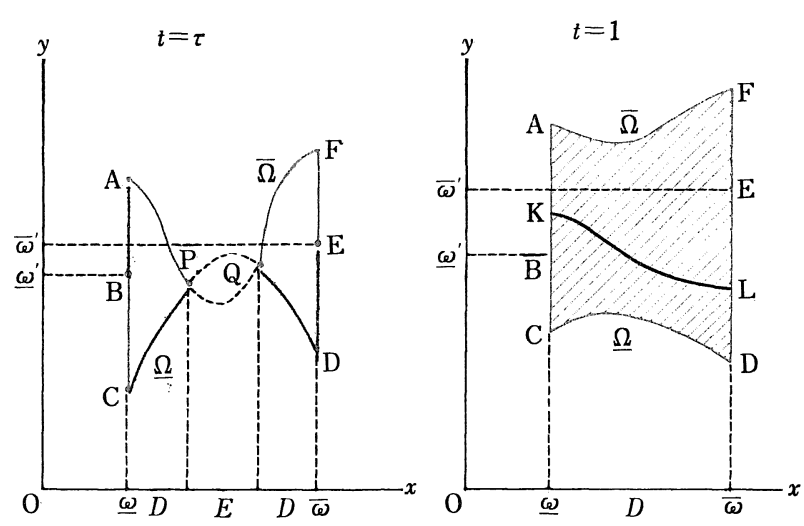

$\mathcal{B}^{+}$

Fig. 4, 5, 6 .

\section{Démonstration de la Proposition 2.}

Considérons le cas où l'expression (13.2) est positive pour $\Omega=\underline{\Omega}$ et négative pour $\Omega=\bar{\Omega}$. 
Considérons les sections $\mathcal{S}_{0}, \mathcal{S}_{1}$ de l'ensemble $\mathscr{D}$ par les plans $t=0,1$ :

$$
\begin{array}{ll}
S_{0}: & \underline{\omega}(0) \leqq x \leqq \bar{\omega}(0), \underline{\Omega}(0, x) \leqq y \leqq \bar{\Omega}(0, x), \\
\mathcal{S}_{1}: & \underline{\omega}(1) \leqq x \leqq \bar{\omega}(1), \underline{\Omega}(1, x) \leqq y \leqq \bar{\Omega}(1, x) .
\end{array}
$$

Prenons un point $(0, \xi, \eta) \in \mathcal{S}_{0}$. Grâce à la condition d'unicité, il n'existe qu'une courbe solution issue du point $(0, \xi, \eta)$. Soit $\left(\tau, \xi^{\prime \prime}, \eta^{\prime \prime}\right)$ son extrémité droite.

Si $\tau=1$, posons $\xi^{\prime}=\xi^{\prime \prime}, \eta^{\prime}=\eta^{\prime \prime}$.

Si $\tau<1, \quad \xi^{\prime \prime}$ est égale à $\underline{\omega}(\tau)$ ou à $\bar{\omega}(\tau)$. On a nécessairement $\underline{\omega}(\tau)<\bar{\omega}(\tau)$, car l'égalité $\underline{\omega}(\tau)=\bar{\omega}(\tau)$ impliquerait $\underline{\omega}^{\prime}(\tau)=\bar{\omega}^{\prime}(\tau)$. On pourrait en déduire, comme au $\mathrm{n}^{\circ}$ précédent, une contradiction avec l'unicité de solution. Posons

$$
\begin{aligned}
\xi^{\prime}=\underline{\omega}(0)+\tau-1, \eta^{\prime} & =\underline{\underline{\omega}} \underline{\prime}^{\prime}(0) \frac{\eta^{\prime \prime}-\underline{\Omega}(\tau, \underline{\omega}(\tau))}{\underline{\omega}^{\prime}(\tau)-\underline{\Omega}(\tau, \underline{\omega}(\tau))} \\
& +\underline{\Omega}(0, \underline{\omega}(0)) \frac{\underline{\omega})^{\prime}(\tau)-\eta^{\prime \prime}}{\underline{\omega}^{\prime}(\tau)-\underline{\Omega}(\tau, \underline{\omega}(\tau))}
\end{aligned}
$$

o11

$$
\begin{aligned}
\xi^{\prime}=\underline{\omega}(0)-\tau+1, \eta^{\prime}= & \bar{\omega}^{\prime}(0) \frac{\bar{\Omega}(\tau, \bar{\omega}(\tau))-\eta^{\prime \prime}}{\bar{\Omega}(\tau, \bar{\omega})(\tau))-\bar{\omega}^{\prime}(\tau)} \\
& +\bar{\Omega}(0, \bar{\omega}(0)) \frac{\eta^{\prime \prime}-\bar{\omega}^{\prime}(\tau)}{\bar{\Omega}(\tau, \bar{\omega}(\tau))-\bar{\omega}^{\prime}(\tau)}
\end{aligned}
$$

suivant que $\xi^{\prime \prime}=\underline{\omega}(\tau)$ ou $\bar{\omega}(\tau)$. En faisant correspondre à un point $(0, \xi, \eta) \in \mathcal{S}_{0}$ le point $\left(0, \xi^{\prime}, \eta^{\prime}\right)$, nous obtenons une application continue $T$ de $\mathcal{S}_{0}$ dans le plan $t=0$. Si $(0, \xi, \eta)$ est un point fixe de cette transformation, la solution telle que

$$
x(0)=\xi, \quad y(0)=\eta
$$

est périodique par rapport à $I$. Soit $T^{\prime}$ la transformation qui fait correspondre au point $(0, \xi, \eta)$ le point $\left(0, \xi^{\prime}-\xi, \eta^{\prime}-\eta\right)$.

Pour montrer l'existence des points fixes, il suffit de calculer le degré topologique $d\left(0, T^{\prime}, \mathcal{S}_{0}\right)$ à l'origine $\mathrm{O}$.

Soit $\mathrm{P}^{\prime}\left(0, \xi^{\prime}, \eta^{\prime}\right)$ le point correspondant de $\mathrm{P}(0, \xi, \eta)$. Nous voulons calculer la variation de l'argument arg $\mathrm{PP}^{\prime}$ du vecteur $\mathrm{PP}^{\prime}$ lorsque 
P décrit le contour ABCDEFA de $\mathcal{S}_{0}$.

Lorsque $\mathrm{P}$ est sur le segment

$\mathrm{BC}:\left\{\begin{array}{l}x=\underline{\omega}(0), \\ \underline{g}(0, \underline{\omega}(0)) \leqq y \leqq \underline{\omega} \underline{\prime}^{\prime}(0),\end{array}\right.$

$\mathrm{PP}^{\prime}$ est le vecteur unité parallèle à l'axe des $x$ et de sens opposé. Lorsque $\mathrm{P}$ est sur le segment

$$
\mathrm{EF}:\left\{\begin{array}{l}
x=\bar{\omega}(0), \\
\bar{\omega}^{\prime}(0) \leqq y \leqq \bar{\Omega}(0, \bar{\omega}(0)),
\end{array}\right.
$$

$\mathrm{PP}^{\prime}$ est le vecteur unité parallèle à l'axe des $x$ et de même sens. La variation de $\arg \mathrm{PP}^{\prime}$ est nulle lorsque $\mathrm{P}$ décrit le segment $\mathrm{BC}$

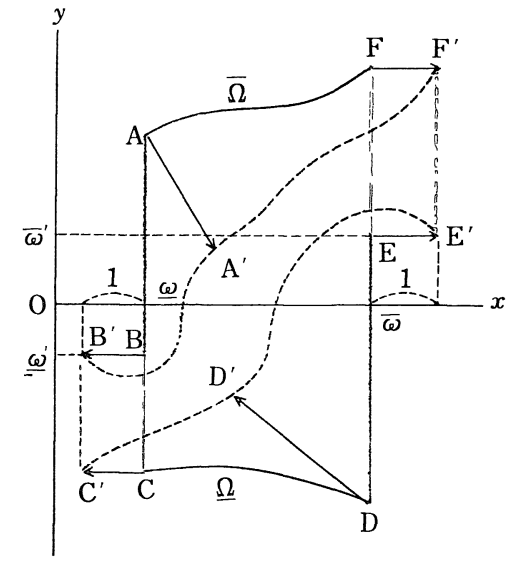

Fig. 7 ou EF.

Lorsque $\mathrm{P}$ décrit la ligne

$\mathrm{CDE}: \quad\left\{\begin{array}{l}\underline{\omega}(0)<x<\bar{\omega}(0), y=\underline{\Omega}(0, x) ; \\ x=\bar{\omega}(0), \underline{\Omega}(0, \bar{\omega}(0)) \leqq y \leqq \bar{\omega}^{\prime}(0),\end{array}\right.$

$\mathrm{P}^{\prime}$ décrit une ligne $\mathrm{C}^{\prime} \mathrm{D}^{\prime} \mathrm{E}^{\prime}$ qui se trouve au dessus de la ligne $\mathrm{C}^{\prime} \mathrm{CDEE}^{\prime}$ et la variation de $\arg \mathrm{PP}^{\prime}$ est égale à $-\pi$. Lorsque $\mathrm{P}$ décrit la ligne

FAB:

$$
\left\{\begin{array}{l}
\bar{\omega}(0)<x<\bar{\omega}(0), y=\bar{\Omega}(0, x) ; \\
x=\underline{\omega}(0), \underline{\omega} \underline{\omega}^{\prime}(0)<y<\bar{\Omega}(0, \underline{\omega}(0)),
\end{array}\right.
$$

$\mathrm{P}^{\prime}$ décrit une ligne $\mathrm{F}^{\prime} \mathrm{A}^{\prime} \mathrm{B}^{\prime}$ qui se trouve au dessous de la ligne $\mathrm{F}^{\prime} \mathrm{FABB}^{\prime}$ et la variation de $\arg \mathrm{PP}^{\prime}$ est égale à $-\pi$.

$\mathrm{La}$ variation totale de arg $\mathrm{PP}^{\prime}$ est donc égale à $-2 \pi$ de sorte que le degré topologique de l'appication $T^{\prime}$ à l'origine $\mathrm{O}$ est égal à -1 .

C.Q.F.D.

On pourra traiter de la même manière le cas où l'expression (13.2) est négative pour $\Omega=\underline{\Omega}$ et positive pour $\Omega=\bar{\Omega}$.

Considérons maintenant le cas où l'expression (13.2) est négative pour $\Omega=\underline{\Omega}$ et $\Omega=\bar{\Omega}$. Il est clair que l'on peut traiter de la même 
manière le cas où l'expression (13.2) est positive pour $\Omega=\underline{\Omega}$ et $\Omega=\bar{\Omega}$.

Comme précédemment, nous désignons par $\left(\tau, \xi^{\prime \prime}, \eta^{\prime \prime}\right)$ l'extrémité droite de la courbe solution issue d'un point $(0, \xi, \eta)$ de $\mathcal{S}_{0}$. Si $\tau=1$. nous posons $\xi^{\prime}=\xi^{\prime \prime}, \eta^{\prime}=\eta^{\prime \prime}$. Si $\tau<1$, trois cas sont à distinguer:

Si $\xi^{\prime \prime}=\bar{\omega}(\tau)$, nous définissons $\xi^{\prime}, \eta^{\prime}$ comme plus haut;

$\mathrm{Si} \xi^{\prime \prime}=\underline{\omega}(\tau)$, nous posons

$$
\begin{aligned}
\xi^{\prime}= & \xi^{\prime \prime}+(\tau-1) \cos \theta\left(\eta^{\prime \prime}\right), \\
\eta^{\prime}= & \underline{\omega}^{\prime}(0) \frac{\eta^{\prime \prime}-\underline{\Omega}(0, \underline{\omega}(\tau))}{\underline{\omega}^{\prime}(\tau)-\underline{\underline{Q}}(\tau, \underline{\omega}(\tau))} \\
& +\underline{\Omega}(0, \underline{\omega}(0)) \frac{\underline{\omega}^{\prime}(\tau)-\eta^{\prime \prime}}{\underline{\omega^{\prime}}(\tau)-, \underline{\underline{\Omega}}(\tau, \underline{\omega}(\tau))}+(\tau-1) \sin \theta\left(\eta^{\prime \prime}\right)
\end{aligned}
$$

où

$$
\theta\left(\eta^{\prime \prime}\right)=\frac{\pi}{2} \frac{\underline{\omega}^{\prime}(\tau)-\eta^{\prime \prime}}{\underline{\omega}^{\prime}(\tau)-\underline{\Omega}(\tau, \underline{\omega}(\tau))} ;
$$

Si $\underline{\omega}(\tau)<\xi^{\prime \prime} \leqq \bar{\omega}(\tau), \eta^{\prime \prime}=\underline{Q}(\tau, \underline{\omega}(\tau))$, nous posons

$$
\xi^{\prime}=\xi^{\prime \prime}, \eta^{\prime}=\underline{\Omega}\left(0, \xi^{\prime \prime}\right)+\tau-1 .
$$

En définissant l'application $T^{\prime}$ comme plus haut, il suffit encore de montrer l'existence d'un point fixe de la transformation $T^{\prime}$.

Or on verra dans ce cas aussi que le degré topologique de la transformation $T^{\prime}$ est égal à -1 . La proposition est donc établie.

Remarque. La première des égalités (13.11) peut être remplacée par

ou

$$
\underline{\Omega}(0, x) \leqq \underline{\Omega}(1, x)
$$

$$
\underline{\Omega}(0, x) \geqq \underline{\Omega}(1, x)
$$

suivant que l'expression (13.2) est positive ou négative pour $\Omega$ $=\underline{\Omega}$, et la deuxième des égalités

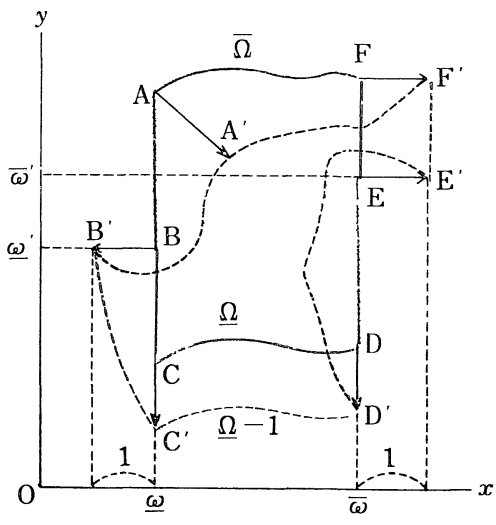

Fig. 8 (13.11) peut être remplacée par 


$$
\bar{\Omega}(0, x) \leqq \bar{\Omega}(1, x) \quad \text { ou } \quad \bar{\Omega}(0, x) \geqq \bar{\Omega}(1, x)
$$

suivant que l'expression (13.2) est positive ou négative pour $\Omega=\bar{\Omega}$. La démonstration reste valable sans aucune modification essentielle.

\section{Démonstration de la Proposition 3 et Son Amélioration.}

Supposons l'existence d'une suite de solutions $\left\{\varphi_{\nu}\right\}$ telles que l'on ait

$$
\varphi_{\nu+1}(t)-\varphi_{\nu}(t)>0
$$

dans $I$. On voit immédiatement que la suite $\left\{\varphi_{\nu}^{\prime \prime}\right\}$ est bornée. Par suite, les suites $\left\{\varphi_{\nu}\right\}$ et $\left\{\varphi_{\nu}^{\prime}\right\}$ convergent respectivement vers une solution $\varphi$ et sa dérivée $\varphi^{\prime}$ uniformément dans $I$. Les conditions $\left(B_{1}\right)$ et $\left(\mathrm{B}_{2}\right)$ sont évidemment remplies.

Considérons la fonction $\psi_{\nu}$ définie par

$$
\psi_{\nu}(t)=c_{\nu}^{-1} \Delta_{\nu}(t)
$$

où

$$
c_{\nu}=\sup \left\{\left|\Delta_{\nu}^{\prime}(t)\right|, \Delta_{\nu}(t) ; t \in I\right\} .
$$

On a évidemment

$$
\sup \left\{\left|\psi_{\nu}^{\prime}(t)\right|, \psi_{\nu}(t) ; t \in I\right\}=1
$$

et $\psi_{\nu}$ est une solution de l'équation différentielle en $u$ :

$$
d^{2} u / d t^{2}=c_{\nu}^{-1}\left\{f\left(t, \varphi(t), \varphi^{\prime}(t)\right)-f\left(t, \varphi(t)-c_{\nu} u, \varphi^{\prime}(t)-c_{\nu} u^{\prime}\right)\right\} .
$$

Si l'on en désigne le second membre par $g_{\nu}\left(t, u, u^{\prime}\right)$, on a

$$
\left|g_{\nu}\left(t, u, u^{\prime}\right)\right| \leqq L|u|+L^{\prime}\left|u^{\prime}\right|
$$

puisqu'on suppose (ii). Par suite, on a

$$
\left|\psi_{\nu}^{\prime \prime}(t) \leqq L\right| \psi_{\nu}(t)\left|+L^{\prime}\right| \psi_{\nu}^{\prime}(t) \mid \leqq L+L^{\prime} .
$$

On peut donc supposer, sans perdre la généralité, que les suites $\left\{\psi_{\nu}\right\}$ et $\left\{\psi_{\nu}^{\prime}\right\}$ convergent respectivement vers une fonctions $\psi$ et sa dérivée $\psi^{\prime}$ uniformément dans $I$.

Si $\psi$ s'annulait identiquement, $\psi^{\prime}$ s'annulerait aussi identiquement puisque $\psi^{\prime}$ est la dérivée de $\psi$. C'est absurde puisque (17.2) entraîne 


$$
\sup \left\{\left|\psi^{\prime}(t)\right|, \psi(t) ; t \in I\right\}=1 .
$$

Par suite, $\psi$ ne s'annule pas identiquement.

Soit $\mu$ la racine positive de l'équation en $\rho$ :

$$
\rho^{2}-L_{\rho}-L^{\prime}=0 \text {. }
$$

D'après un théorème de comparaison bien connu, on a

$$
\left|\psi_{\nu}(t)\right| \leqq B_{\nu} e^{\mu|t-\tau|},\left|\psi_{\nu}^{\prime}(t)\right| \leqq \mu B_{\nu} e^{\mu|t-\tau|}
$$

pour $t, \tau \in I$, où

$$
B_{\nu}=\max \left\{\left|\psi_{\nu}(\tau)\right|,\left|\psi_{\nu}^{\prime}(\tau)\right| / \mu\right\} .
$$

Si donc $\left\{\psi_{\nu}(\tau)\right\}$ et $\left\{\psi_{\nu}^{\prime}(\tau)\right\}$ convergeait vers $0, \psi$ deviendrait identiquement nulle. Par suite, il n'y a aucune valeur $\tau \in I$ où l'on $a \grave{a}$ la fois $\psi(\tau)=0, \psi^{\prime}(\tau)=0$.

Puisque $\psi_{\nu}(t)>0$ pour $t \in I, \psi(\tau)=0$ exigerait $\psi^{\prime}(\tau)=0$ si $0<\tau<$ 1. Par suite $\psi(t)$ est positive pour $0<t<1$.

Il nous reste à montrer que l'on peut choisir la suite $\left\{\varphi_{\nu}\right\}$ de manière que les deux suites

$$
\left\{\Delta_{\nu}^{\prime}(0) / \Delta_{\nu}(0)\right\},\left\{-\Delta_{\nu}^{\prime}(1) / \Delta_{\nu}(1)\right\}
$$

restent bornée supérieurement.

Soit $\theta_{0}$ une fonction linéaire de $x$ qui prend les valeurs

$$
\frac{\beta_{0}-\underline{\Omega}(0, \underline{\omega}(0))}{\bar{\Omega}(0, \underline{\omega}(0))-\underline{\Omega}(0, \underline{\omega}(0))}, \quad \frac{\beta_{0}^{\prime}-\underline{\Omega}(0, \bar{\omega}(0))}{\bar{\Omega}(0, \bar{\omega}(0))-\underline{\Omega}(0, \bar{\omega}(0))}
$$

respectivement pour $x=\underline{\omega}(0), \bar{\omega}(0)$ et posons

$$
h(x)=\theta_{0}(x) \bar{\Omega}(0, x)+\left(1-\theta_{0}(x)\right) \underline{\Omega}(0, x),
$$

où $\beta_{0}$ et $\beta_{0}^{\prime}$ sont des valeurs telles que

$$
\underline{\Omega}(0, \underline{\omega}(0))<\beta_{0}<\underline{\omega}^{\prime}(0), \bar{\omega}^{\prime}(0)<\beta_{0}^{\prime}<\bar{\Omega}(0, \bar{\omega}(0)) .
$$

On a évidemment

$$
0<\theta_{0}(x)<1, \underline{\Omega}(0, x)<h(x)<\bar{\Omega}(0, x)
$$

pour $\underline{\omega}(0) \leqq x \leqq \bar{\omega}(0)$ et

$$
h(\underline{\omega}(0))=\beta_{0}, h(\bar{\omega}(0))=\beta_{0}^{\prime} .
$$


Nous définissons de même une fonction $k \in \mathrm{C}^{1}$ telle que l'on ait

$$
\underline{\Omega}(1, x)<k(x)<\bar{\Omega}(1, x)
$$

pour $\underline{\omega}(1) \leqq x \leqq \bar{\omega}(1)$ et

$$
k(\underline{\omega}(1))=\beta_{1}, k(\bar{\omega}(1))=\beta_{1}^{\prime},
$$

où $\beta_{1}, \beta_{1}^{\prime}$ sont des valeurs telles que l'on ait

$$
\underline{\omega}^{\prime}(1)<\beta_{1}<\bar{\Omega}(1, \underline{\omega}(1)), \underline{\Omega}(1, \bar{\omega}(1))<\beta_{1}^{\prime}<\bar{\omega}^{\prime}(1) .
$$

Prenons d'autre part une suite décroissante $\left\{\varepsilon_{\nu}\right\}$ convergeant vers 0 . Nous supposons $\varepsilon_{1}$ assez petit de sorte que l'on a

$$
h(x)+\varepsilon_{1}<\bar{\Omega}(0, x)
$$

pour $\underline{\omega}(0) \leqq x \leqq \bar{\omega}(0)$,

$$
\underline{Q}(1, x)<k(x)-\varepsilon_{1}
$$

pour $\underline{\omega}(1) \leqq x \leqq \bar{\omega}(1)$, et

$$
\beta_{0}+\varepsilon_{1}<\underline{\omega}^{\prime}(0), \underline{\omega}^{\prime}(1)<\beta_{1}^{\prime}-\varepsilon_{1} .
$$

Posons ensuite

$$
h_{\nu}(x)=h(x)+\varepsilon_{\nu}, k_{\nu}(x)=k(x)-\varepsilon_{\nu} .
$$

Soit $\mathcal{E}_{1}$ la courbe

$$
t=0, \underline{\omega}(0) \leqq x \leqq \bar{\omega}(0), y=h_{1}(x)
$$

et $\mathcal{E}_{1}^{\prime}$ la courbe

$$
t=1, \underline{\omega}(1) \leqq x \leqq \bar{\omega}(1), y=k_{1}(x) .
$$

D'après notre théorème d'existence, il existe une solution $\varphi_{1}$ dont le graphique joint $\mathcal{E}_{1}$ à $\mathcal{E}_{1}^{\prime}$.

Soit $\mathcal{E}_{2}$ la courbe

$$
t=0, \varphi_{1}(0) \leqq x \leqq \bar{\omega}(0), y=h_{2}(x)
$$

et $\mathcal{E}_{2}^{\prime}$ la courbe

$$
t=1, \varphi_{1}(1) \leqq x \leqq \bar{\omega}(1), y=k_{2}(x) .
$$

Nous pouvons appliquer notre théorème d'existence, en remplaçant $\underline{\omega}$ $\operatorname{par} \varphi_{1}$. Il existe donc une solution $\varphi_{2}$ dont le graphique joint $\mathcal{E}_{2} \grave{a} \mathcal{E}_{2}^{\prime}$. 
Soit $\mathcal{E}_{3}$ la courbe

$$
t=0, \varphi_{2}(0) \leqq x \leqq \bar{\omega}(0), y=h_{3}(x)
$$

et $\mathcal{E}_{3}^{\prime}$ la courbe

$$
t=1, \varphi_{2}(1) \leqq x \leqq \bar{\omega}(1), y=k_{3}(x) .
$$

Nous pouvons appliquer notre théorème d'existence, en remplaçant $\underline{\omega}$ par $\varphi_{2}$. Il existe donc une solution $\varphi_{3}$ dont le graphique joint $\mathcal{E}_{3}$ à $\mathcal{E}_{3}^{\prime}$, et ainsi de suite.

Soit $\mathcal{E}$ la courbe

$$
t=0, \underline{\omega}(0) \leqq x \leqq \bar{\omega}(0), y=h(x)
$$

et $\mathcal{E}^{\prime}$ la courbe

$$
t=1, \underline{\omega}(1) \leqq x \leqq \bar{\omega}(1), y=k(x) .
$$

$\varphi$ est une solution dont le graphique joint $\mathcal{E}$ à $\mathcal{E}^{\prime}$.

Nous obtenons ainsi une suite de solutions $\left\{\varphi_{\nu}\right\}$, et les conditions $\left(B_{1}\right)$ et $\left(B_{2}\right)$ sont remplies.

Si l'on pose

$$
\xi=\varphi(0), \xi_{\nu}=\varphi_{\nu}(0)
$$

on a

$$
\Delta_{\nu}(0)=\xi-\xi_{\nu}, \Delta_{\nu}^{\prime}(0)=h(\xi)-h\left(\xi_{\nu}\right)-\varepsilon_{\nu} .
$$

On a donc

$$
\frac{\Delta_{\nu}^{\prime}(0)}{\Delta_{\nu}(0)} \leqq \frac{h(\xi)-h\left(\xi_{\nu}\right)}{\xi-\xi_{\nu}} \leqq \sup \left\{h^{\prime}(x) ; \underline{\omega}(0) \leqq x \leqq \bar{\omega}(0)\right\} .
$$

On verra de même que la suite $\left\{-\Delta_{\nu}^{\prime}(1) / \Delta_{\nu}(1)\right\}$ est bornée. La solution $\varphi$ jouit donc de la propriété $\left(B_{3}\right)$ et la proposition 3 est démontrée.

$\varphi$ étant une solution dont le graphique joint $\mathcal{E}$ à $\mathcal{E}^{\prime}$, on a non seulement les inégalités (13.12), (13.13) mais aussi les inégalités strictes (13.6), (13.7).

$\psi$ ne s'annulant jamais dans $I$, sa valeur minimum $\delta$ est positive. $\left|\psi^{\prime}\right|$ ne pouvant surpasser 1 , on a la condition $\left(B_{3}^{\prime}\right)$.

On a donc la proposition 3 améliorée:

Si toutes les hypothèses de la proposition 1 sont remplies, il 
existe une solution $\varphi$ satisfaisant aux conditions $\left(\mathrm{B}_{1}\right),\left(\mathrm{B}_{2}\right)$ et $\left(\mathrm{B}_{3}^{\prime}\right)$ et aux inégalités strictes (13.6), (13.7).

\section{Bibliographie}

H. W. Knobloch,

[1] Comparison theorems for nonlinear second order differential equations, J. Differ. Eqns. 1 (1965), 1-26.

[2] Eine neue Methode zur Approximation periodischer Lösungen nicht-linearer Differentialgleichungen zweiter Ordnung, Math. Z. 82 (1963), 177-197.

M. Nagumo,

[1] Über die Differentialgleichung $y^{\prime \prime}=f\left(x, y, y^{\prime}\right)$. Proc. Phys.-Math. Soc. Japan, III, 19 (1937), 861-866.

[2] Dai-2-kai Zyôbibunhôteisiki no Kyôkaiti Mondai, I, II, Kansû Hôteisiki, No. 5 (1939), 27-34; No. 6 (1939), 27-44.

[3] Über das Randwertproblem der nicht linearen gewöhnlichen Differentialgleichungen zweiter Ordnung, Proc. Phys.-Math. Soc. Japan. III, 24 (1942), 845851.

\section{Errata à la partie précédente}

\begin{tabular}{|c|c|c|c|}
\hline Page & Ligne & Au lieu de & Lire \\
\hline \multirow[t]{2}{*}{247} & 18 & $Z^{+}(E)$ & $3^{+}(\mathcal{E})$ \\
\hline & 6 en remontant & est & soit \\
\hline 249 & 15 & appartenant à $\mathfrak{A}_{1}$ & appartenant à $\mathfrak{U}_{2}$ \\
\hline 250 & 3 & demonstration & démonstration \\
\hline \multirow[t]{4}{*}{251} & 18 & $\mathbb{F}_{\alpha_{k}}^{+}\left(P_{i}^{\prime}\right)$ & $\hat{\boldsymbol{F}}_{\alpha_{k}}^{+}\left(P_{i}^{\prime}\right)$ \\
\hline & & $F_{\alpha_{k}}^{+}\left(P_{i+1}^{\prime}\right)$ & $\hat{\boldsymbol{F}}_{\alpha_{k}}^{+}\left(P_{i+1}^{\prime}\right)$ \\
\hline & 10 en remontant & $Q_{m}^{\prime}, Q_{m}^{\prime \prime}$ & $P_{m}^{\prime}, Q_{m}^{\prime \prime}$ \\
\hline & 6 en remontant & $Q_{i}^{\prime}$ et $P_{i+1}^{\prime}$ & $P_{i}^{\prime}$ et $P_{i+1}^{\prime}$ \\
\hline \multirow[t]{2}{*}{258} & 11 en remontant & $S$ & $\mathfrak{S}$ \\
\hline & 4 en remontant & $B\left(\mathbb{F}_{\mathscr{S}}^{g}\right)$ & $\mathfrak{B}\left(\boldsymbol{F}_{\mathscr{S}}^{g}\right)$ \\
\hline 259 & 6 en remontant & $(t, \mathfrak{x}(t)]$ & $(t, \mathfrak{x}(t))$ \\
\hline \multirow[t]{2}{*}{260} & 9 en remontant & $\mathfrak{S}=\mathfrak{G} \cap\left(\mathfrak{B}^{d} \cup \mathfrak{B}_{+}\right)$ & $\mathfrak{F} \cap\left(\mathfrak{B}^{d} \cup \mathfrak{B}_{+}\right)$ \\
\hline & 7 en remontant & $3^{+}(\xi)$ & $\boldsymbol{F}^{+}(\mathfrak{\xi})$ \\
\hline \multirow[t]{2}{*}{262} & 11 & $\geqq 0$ & $\leqq 0$ \\
\hline & 12 & $\leqq 0$ & $\geqq 0$ \\
\hline 265 & 1 en remontant & $3_{\tau+\delta}^{+}\left(A, \boldsymbol{F}^{\prime}\right)$ & $3_{\tau-\delta}^{-}\left(A, \boldsymbol{F}^{\prime}\right)$ \\
\hline
\end{tabular}


Page 263. Remplacer la démonstration de la proposition 5.3 par la suivante:

Il suffit de montrer qu'un point $A \notin \mathfrak{B}^{d}$ est knesérien à droite.

Soit $\tau$ une valeur quelconque plus grande que l'abscisse de $A$. Si l'extrémité droite de $\mathfrak{x} \in \hat{\boldsymbol{F}}^{+}(A)$ est à gauche de l'hyperplan $t=\tau$, nous posons $\varphi(\mathfrak{x})=\mathfrak{x}$. Sinon nous désignons par $\varphi(\mathfrak{x})$ la partie de $\mathfrak{x}$ limitée par les hyperplans $t=\alpha, t=\tau$. $\varphi$ est alors une application continue de $\widehat{\boldsymbol{F}}^{+}(A)$ sur $\widehat{\boldsymbol{F}}_{\tau}^{+}(A)$, ensemble des caractéristiques limitées par $A$ et

$$
\mathfrak{S}=\mathfrak{S} \cup\left(\mathfrak{Z}_{\tau}^{+}(A) \cap\left(\mathfrak{B}_{+} \cup \mathfrak{B}^{d}\right)\right) .
$$

$\boldsymbol{F}^{+}(A)$ étant un continu, $\hat{\boldsymbol{F}}_{\tau}^{+}(A)$ l'est aussi.

Si l'on fait correspondre à une caractéristique de $\widehat{\mathbb{F}}_{\tau}^{+}(A)$ son extrémité droite, on obtient une application continue de $\widehat{\mathbb{F}}_{\tau}^{+}(A)$ sur $\mathfrak{c}$. Par suite $\sqrt{ }$ est un continu et $A$ est un point knesérien à droite.

Page 268. Remplacer la figure par la suivante.

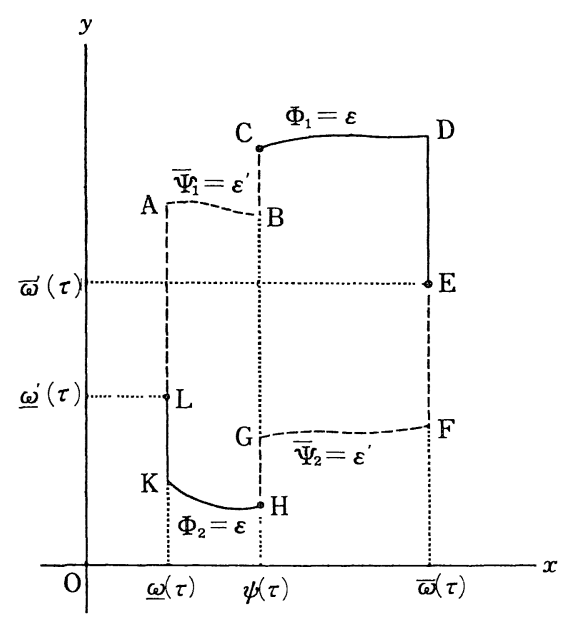


